\title{
Structural and Functional Analysis of Keratinicyclin Reveals Syner- gistic Antibiosis with Vancomycin against Clostridium difficile
}

\author{
Vasiliki T. Chioti, ${ }^{\dagger} \&$ Kirklin L. McWhorter, ${ }^{\ddagger}, \&$ Fei Xu, ${ }^{\wedge}$ Philip D. Jeffrey, ${ }^{\#}$ Katherine M. Davis,,$* *$ and \\ Mohammad R. Seyedsayamdost $\mathrm{t}^{\dagger, \#, *}$ \\ ${ }^{\dagger}$ Department of Chemistry, Princeton University, Princeton, New Jersey 08544, United States \\ *Department of Chemistry, Emory University, Atlanta, Georgia, 30322, United States \\ ^Institute of Pharmaceutical Biotechnology, Zhejiang University School of Medicine, Hangzhou 310058, China

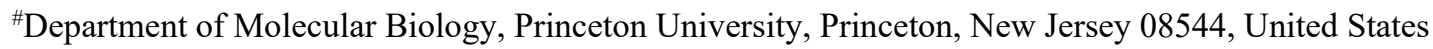 \\ ${ }^{\&}$ These authors contributed equally and are listed alphabetically
}

\section{Supporting Information Placeholder}

\begin{abstract}
Keratinicyclins and keratinimicins are recently discovered glycopeptide antibiotics (GPAs). The latter are canonical GPAs with broad-spectrum activity against Gram-positive bacteria, while keratinicyclins form a new chemotype by virtue of an unusual oxazolidinone moiety and exhibit specific antibiosis against Clostridium difficile. Here, we investigated the three-dimensional structures and functional consequences for both molecules. Equilibrium binding studies showed tight binding by keratinimicin A, but not keratinicyclin B, to the peptidoglycan terminus. Using protein crystallography methods, we solved the X-ray crystal structures of both GPAs, which, in conjunction with DFT calculations, indicate that the inability of keratinicyclin B to bind the peptidoglycan is governed by steric factors. Keratinicyclin B, therefore, interferes with an alternative target to inhibit $C$. difficile growth, a conclusion confirmed by checkerboard analysis that revealed synergistic activity with vancomycin. Our results set the stage for identifying the molecular target of keratinicyclins and for exploring their therapeutic utility in combination with vancomycin.
\end{abstract}

Glycopeptide antibiotics (GPAs) have been indispensable in the fight against infectious disease. ${ }^{1-4}$ To date, over a hundred members have been characterized giving rise to at least five different structural classes. Mode of action studies, many conducted before the structure of the founding member vancomycin (1, Fig. 1) was solved, ${ }^{5-8}$ have identified the growing peptidoglycan chain as the target of $\mathbf{1}$. Specifically, five H-bonds have been shown to be essential in binding the D-Ala-D-Ala terminus of the peptidoglycan. Aside from uncovering additional biological targets, the discovery of new GPAs provides opportunities to study the relationship between structure and function as well as the mechanisms underlying molecular recognition. ${ }^{9}$ Keratinimicins and keratinicyclins are two such new analogs. ${ }^{10}$ Keratinimicin A (2) is a class II GPA that exhibits broad-spectrum activity against diverse Gram-positive bacteria. Keratinicyclins, on the other hand, form a new chemotype within the GPAs owing to the presence of an unusual $N$-terminal oxazolidinone. Variant B (3) harbors antiviral properties and only specific antimicrobial activity against Clostridium difficile. ${ }^{10}$ Both $\mathbf{2}$ and $\mathbf{3}$ were identified following activation of a silent biosynthetic gene cluster in Amycolatopsis keratiniphila, and their structures were elucidated by extensive NMR analysis. Two aspects, however, remain unresolved. Absolute configurations of stereogenic centers were inferred from bioinformatic analyses, but have not been experimentally determined. Moreover, the lack of broad antibacterial activity for $\mathbf{3}$, despite containing all H-bonding components to bind D-Ala-D-Ala, remains unexplained. Here, we address these topics using a combination of experimental and computational studies.
To explore whether lack of peptidoglycan binding by $\mathbf{3}$ explains its narrow-spectrum antimicrobial phenotype, we carried out equilibrium binding studies using isothermal titration calorimetry (ITC). As control, we first tested binding of $\mathbf{1}$ to the $N \alpha, N \varepsilon$-diacetyl-L-Lys-D-Ala-D-Ala tripeptide - the terminal three residues of a typical Gram-positive peptidoglycan - and observed formation of a $1: 1$ complex with a $K_{\mathrm{d}}$ of $2.7 \pm 0.4 \mu \mathrm{M}$ as well as $\Delta \mathrm{H}$ and $\mathrm{T} \Delta \mathrm{S}$ of $-8.6 \pm 0.5 \mathrm{kcal} / \mathrm{mol}$ and -0.96 $\mathrm{kcal} / \mathrm{mol}$, respectively (Fig. 2A). Both the affinity and entropic penalty for binding the tripeptide are consistent with previous results for vancomycin. ${ }^{11}$ Ligand binding induces back-to-back dimerization of $\mathbf{1}$ via intermolecular H-bonds. ${ }^{12,13}$ However, we did not probe cooperativity under our ITC conditions, as the operating concentrations were below the equilibrium constant for dimerization. With 2 , we also observed formation of a $1: 1 \mathrm{com}-$ plex, this time with a $K_{\mathrm{d}}$ of $1.7 \pm 0.3 \mu \mathrm{M}, \Delta \mathrm{H}$ of $7.0 \pm 0.3$ $\mathrm{kcal} / \mathrm{mol}$ and $\mathrm{T} \Delta \mathrm{S}$ of $0.85 \mathrm{kcal} / \mathrm{mol}$ (Fig. $2 \mathrm{~B}$ ). Thus, while $\Delta \mathrm{G}$ for the interaction is similar for $\mathbf{1}$ and $\mathbf{2}$, binding by $\mathbf{2}$ is both enthalpically and entropically driven. We suspect that the entropic gain with 2 stems from increased solvent disorder upon ligand binding as keratinimicin is significantly more hydrophobic than 1. Keratinicyclin B, on the other hand, did not bind the tripeptide at several concentrations tested despite the presence of the five canonical H-bonding moieties (Fig. 2C). Based on the sensitivity of our set-up, we estimate a $K_{\mathrm{d}}>0.5 \mathrm{mM}$.

Extensive studies by Perkins, Williams, and others have defined the molecular basis for interaction between $\mathbf{1}$ and its target. $^{1-8}$ Collectively, they suggest three hypotheses that may explain why 3 does not bind the tripeptide. (i) The charged $N$-ter- 


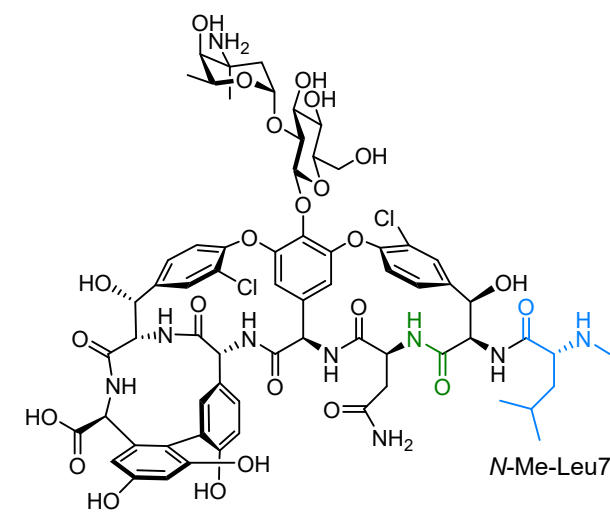

Vancomycin (1)

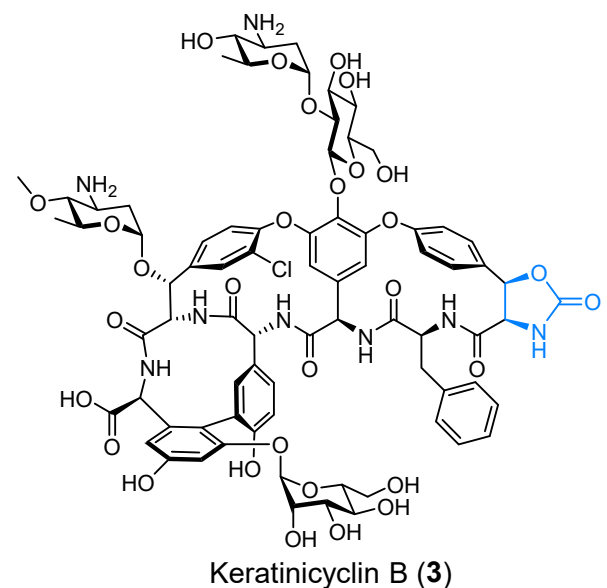

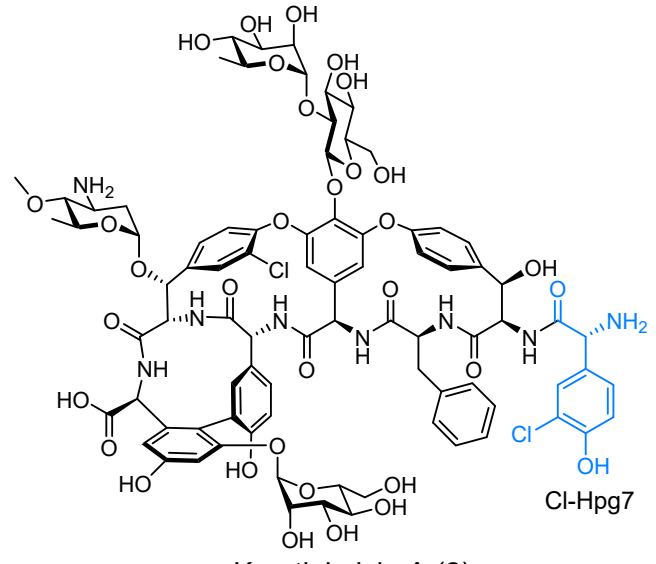

Keratinimicin A (2)

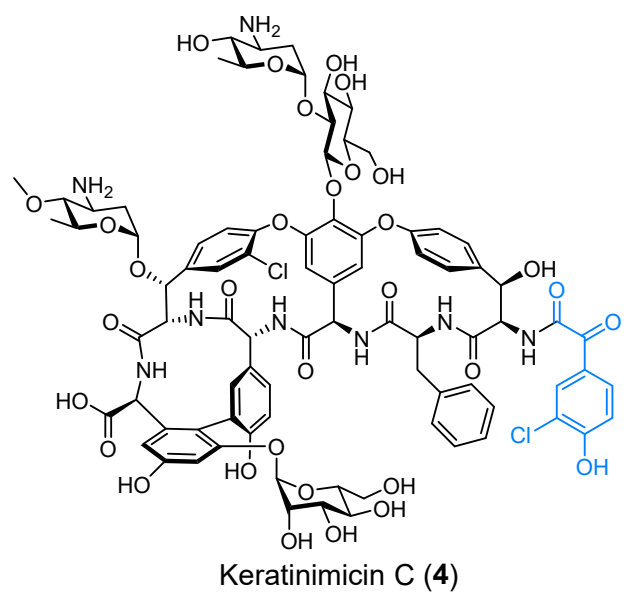

Figure 1. Structures of GPAs studied herein. The seventh residue is shown in blue, where keratinicyclin B instead contains an oxazolidinone. The amide that is flipped out in a 6mer vancomycin is shown in green.
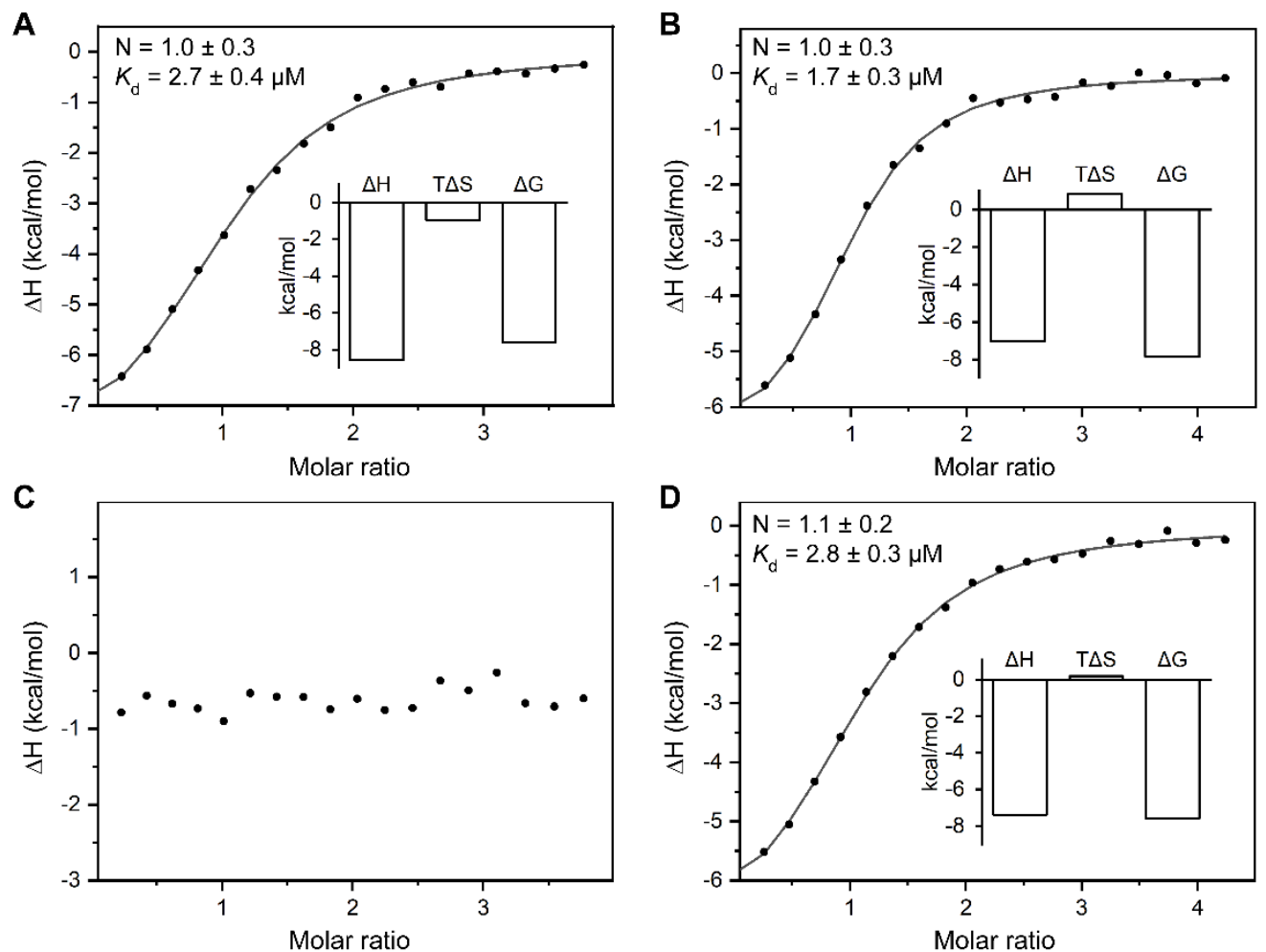

Figure 2. Binding of GPAs to the tripeptide ligand. Shown are ITC-derived binding isotherms for 1 (A), 2 (B), 3 (C), and 4 (D). Complex stoichiometry $(\mathrm{N}), K_{\mathrm{d}}, \Delta \mathrm{H}, \mathrm{T} \Delta \mathrm{S}$, and $\Delta \mathrm{G}$ for the interaction are given in each plot. 
minal ammonium group of $\mathbf{1}$ is known to be important for ligand binding, accounting for $1.5-2 \mathrm{kcal} / \mathrm{mol}$ of the interaction. ${ }^{14}$ In contrast to 2 , which contains a charged amino terminus at neutral $\mathrm{pH}$, the oxazolidinone of $\mathbf{3}$ is uncharged. (ii) Williams and colleagues have shown that the 6 mer version of 1 , in which the $N$-terminal $N$-Me-D-Leu has been removed by Edman degradation $\left(\mathbf{1}^{\prime}\right)$, does not bind the tripeptide ligand. ${ }^{15}$ This has been attributed to disruption of the position of the residue 6 amide in 1 (Fig. 1), which flips out of the binding pocket thereby precluding or weakening the interaction with the tripeptide. Finally, (iii) Perkins demonstrated that the binding pocket for the peptide ligand is rigid and small; ${ }^{6}$ sterics are therefore important for the interaction, a conclusion that has been verified by structural studies of the vancomycin-tripeptide complex. ${ }^{7,8}$

To test hypothesis ( $i$ ) above, we turned to keratinimicin C (4), a natural derivative of $\mathbf{2}$ in which the $N$-terminal amine is replaced with a ketone, thereby removing the positive charge at this position. ${ }^{10}$ ITC binding studies with 4 yielded a $K_{\mathrm{d}}$ of $2.8 \pm$ $0.3 \mu \mathrm{M}$ as well as $\Delta \mathrm{H}$ and $\mathrm{T} \Delta \mathrm{S}$ of $-7.4 \pm 0.3 \mathrm{kcal} / \mathrm{mol}$ and 0.2 $\mathrm{kcal} / \mathrm{mol}$, respectively, similar parameters as 2 (Fig. 2D). This result is consistent with the analogous bioactivity profiles of $\mathbf{2}$ and $4 .{ }^{10}$ Thus, the lack of positive charge at the oxazolidinone of $\mathbf{3}$ does not explain its inability to bind the target peptide.

Next, we explored the topology of the two GPAs and assessed the second hypothesis by determining structures of $\mathbf{2}$ and $\mathbf{3}$ using X-ray crystallography. Repeated attempts to crystallize both compounds via small molecule crystallization methods failed. However, using vapor diffusion techniques typically employed for proteins, we successfully generated crystals of $\mathbf{2}$ and $\mathbf{3}$ suit- able for X-ray diffraction analysis. Their structures were subsequently solved to $0.95 \AA$ resolution (Table S1), thus providing information regarding the backbone conformation and the absolute configuration at chiral centers (Fig. 3, Fig. S1-S2).

The crystal structure of 2 reveals a typical cup-shaped topology, the backbone configuration of which aligns well with the structure of 1 (PDB accession 1FVM, chain A rmsd of $0.36 \AA$ over 28 atoms, Fig. 3A-C) ${ }^{8}$ Intriguingly, we observed formate and chloride ions derived from the crystallization conditions, occupying similar sites as the terminal carboxyl group and an amide of the tripeptide, when compared to the complexed vancomycin crystal structure, supportive of an analogous binding mode. The structure of $\mathbf{2}$ confirms the absolute configurations we proposed based on NMR and bioinformatic analyses of the ker gene cluster. ${ }^{10}$ Crystallographic data obtained for $\mathbf{3}$ likewise verify the absolute configurations proposed in the original structural studies (Fig. 3D). They further reveal the oxazolidinone in near perpendicular arrangement with respect to the plane created by the central aryl-ether-crosslinked aromatic sidechains. Alignment of $\mathbf{2}$ and $\mathbf{3}$ shows that their backbone conformations are almost identical with rmsds between 0.14$0.25 \AA$ over 24 atoms when comparing equivalent chains (Fig. $3 \mathrm{E}$, Movie S1). Indeed, the position of the H-bonding partners that are important in binding the tripeptide are entirely homologous, and the central amide of residue 6 is not flipped away from the binding site. It thus seems unlikely that hypothesis (ii) explains the disparate tripeptide binding properties of $\mathbf{2}$ and $\mathbf{3}$.

The experiments thus far rule out explanation (i) and (ii). In order to evaluate the impact of sterics and the third hypothesis
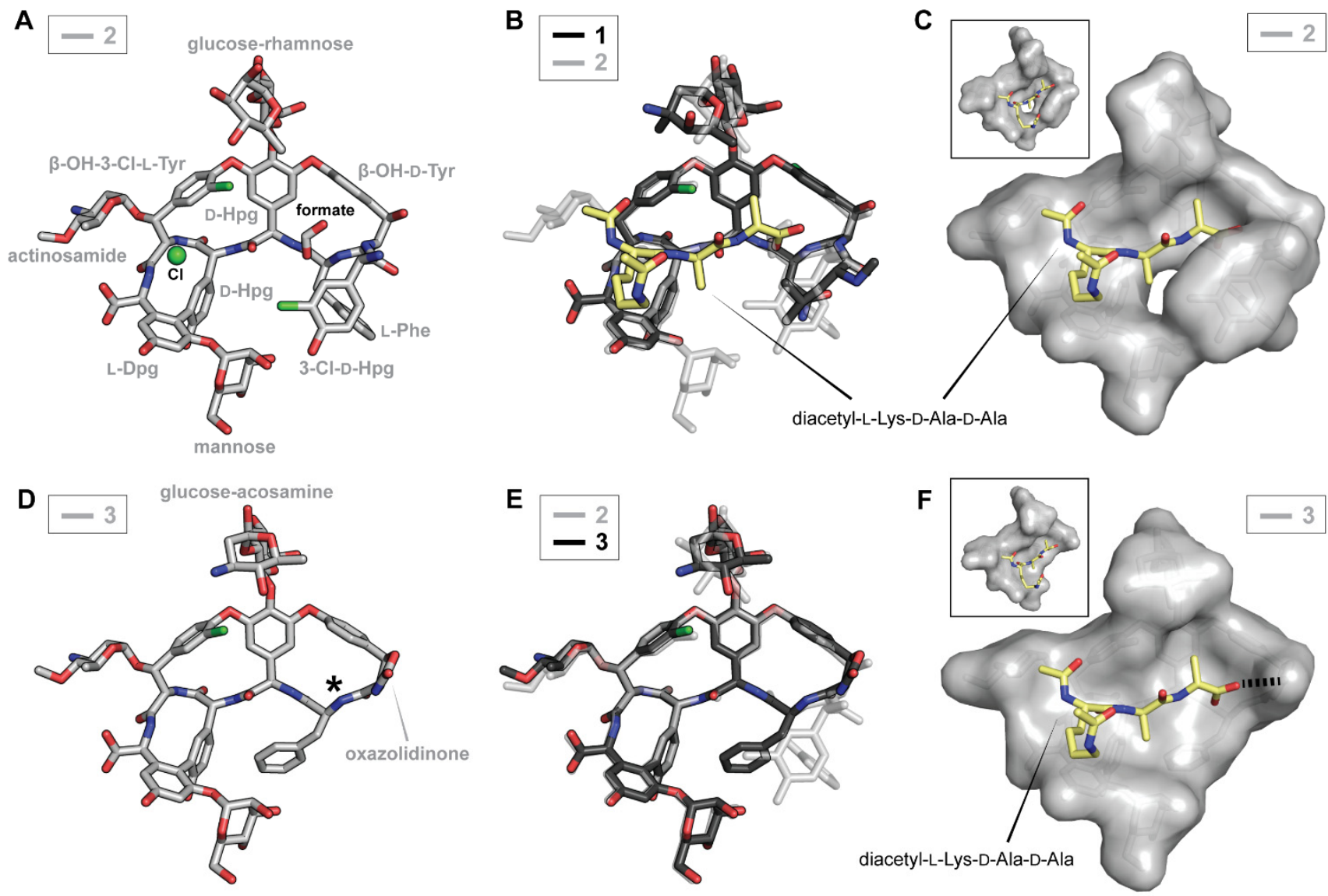

Figure 3. X-ray crystal structures of $\mathbf{2}$ and 3. (A) Structure of 2; amino acids, formate, and chloride are labeled. (B) Overlay of the structures of 1 and 2. (C) Space-filling model of 2 bound to the tripeptide. The inset shows the view of the complex from above. (D) Crystal structure of 3. The peptide bond that flips out in compound 1' is marked (*). (E) Overlay of the structures of 2 and 3. (F) Space-filling model of $\mathbf{2}$ bound to the tripeptide. The inset shows the view of the complex from above. Dashed line highlights possible O-O repulsion between 3 and the ligand. Details regarding crystallographic dimers of $\mathbf{2}$ and $\mathbf{3}$ are outlined in the SI (Fig. S1-S2). 
outlined above, we used the position of the formate and chloride and the overall structural congruence of $\mathbf{1}, \mathbf{2}$, and $\mathbf{3}$ to generate a model of the tripeptide complex of $\mathbf{2}$ and $\mathbf{3}$ (Figs. 3C, 3F). The resulting space-filling models highlight the importance of $\mathrm{Cl}$ Hpg7 in creating a small rigid binding pocket that locks the tripeptide ligand in place. This steric lock is missing in $\mathbf{3}$, which may explain the different affinities of $\mathbf{2}$ and $\mathbf{3}$ for the tripeptide. Moreover, the model shows a short distance $(\sim 3.6 \AA)$ between the oxazolidinone carbonyl-oxygen and the terminal carboxyl group of the ligand (Fig. 3F), possibly causing a repulsive interaction. This distance is similar to the $\mathrm{O}-\mathrm{O}$ repulsion that render bacteria, which incorporate D-Ala-D-lactate, resistant to 1. $^{16-18}$ In the absence of simple experiments to test the importance of steric and stereoelectronic effects, we resorted to DFT-based calculations, in which we sought to estimate the contribution toward binding energy provided by $\mathrm{Cl}-\mathrm{Hpg} 7$ in 2 .

DFT optimizations were performed at the B3LYP/6-31G** level, selected to more accurately describe H-bonding interactions. ${ }^{19,20}$ Frequency calculations provided thermochemical values and ensured an energetic minimum; use of the polarizable continuum model helped simulate the electrostatic effects of water. ${ }^{21}$ Using Avagadro, hydrogens were added to models of the tripeptide-complex of $\mathbf{2}$ and $\mathbf{3}$ assuming neutral $\mathrm{pH} \cdot{ }^{22,23}$ The resultant complexes were then energy-minimized with the $\mathrm{UFF}^{24}$ forcefield to help with steric clashes brought on by the alignment procedure, before DFT optimization. We also computed the binding energy of desleucyl-1 (1'), which does not appreciably bind the tripeptide $\left(K_{\mathrm{d}} \sim 20 \mathrm{mM}\right),{ }^{15}$ and a variant of 3 in which the carbonyl-oxygen of the oxazolidinone is replaced with an $\mathrm{NH}$ group $\left(\mathbf{3}^{\prime}\right)$ to test the effect of O-O repulsion.

Binding energies, enthalpies, and Gibbs free energies were computed for various GPA-ligand complexes (Table 1, Tables $\mathrm{S} 2-\mathrm{S} 6)$. As is typical, the calculated $\Delta \mathrm{G}$ values differ from those obtained experimentally, because only a single optimized conformer is sampled in calculations whereas many microstates are averaged in solution. Computationally-derived binding energies for $\mathbf{1}$ and $\mathbf{1}$ ' were consistent with reported experimental results in which the latter is effectively unable to bind the tripeptide. ${ }^{15}$ The calculated affinity of $\mathbf{3}$ for the tripeptide ligand was 6.4 $\mathrm{kcal} / \mathrm{mol}$ lower than that of $\mathbf{2}$. Thus, despite the presence of the five H-bonding partners in suitable orientations, the calculations similarly reinforce the inability of $\mathbf{3}$ to bind the tripeptide. The 7 mer backbones of $\mathbf{1}$ and $\mathbf{2}$ form a cup-shaped structure that engulfs the tripeptide. This topology is not formed in 3. Moreover, the computed affinity of $\mathbf{3}^{\prime}$ was unchanged relative to $\mathbf{3}$, suggesting that the inability to bind the tripeptide is largely governed by steric factors.

Previous bioactivity data with $\mathbf{3}$ show that it inhibits $C$. difficile growth with a minimal inhibitory concentration (MIC) of $9.8 \mu \mathrm{M} .{ }^{10}$ In light of the results above, the bioactivity data suggest that 3 interferes with an alternative target, rather than the peptidoglycan terminus, to inhibit growth. If $\mathbf{3}$ interferes with an alternative target, synergistic interaction between $\mathbf{1}$ and $\mathbf{3}$ would be expected. We carried out checkerboard analysis ${ }^{25-27}$ in which the MIC of $\mathbf{1}$, or $\mathbf{3}$, or a combination of $\mathbf{1}$ and $\mathbf{3}$ was determined against $C$. difficile and a fractional inhibitory concentration index was computed (see SI). With 1 alone, we determined an MIC of $1 \mu \mathrm{g} / \mathrm{mL}$, consistent with previous results. With 3 alone, we recorded an MIC of $4 \mu \mathrm{g} / \mathrm{mL}$. The combination of 1 and $\mathbf{3}$ clearly indicated a synergistic interaction at several concentrations (Fig. 4). Most notably, mere titers of 0.25 $\mu \mathrm{g} / \mathrm{mL}$ of 1 and $0.5 \mu \mathrm{g} / \mathrm{mL}$ of $\mathbf{3}$ were sufficient to completely inhibit the growth of $C$. difficile. By contrast, 1 and teicoplanin, a well-known D-Ala-D-Ala binder, ${ }^{1-4}$ exhibited only additive interactions (Fig. S3). These results are consistent with the lack of D-Ala-D-Ala binding by $\mathbf{3}$ and validate an alternative, possibly new molecular target, which may be used in the future to identify new $C$. difficile antibiotics.

Table 1. Calculated binding energies $(\mathrm{kcal} / \mathrm{mol})$ between the GPA indicated and the tripeptide ligand. ${ }^{a}$

\begin{tabular}{c|c|c|c|c}
\hline \multicolumn{5}{c}{ Calculation } \\
\hline $\mathbf{G P A}$ & $\mathbf{\Delta E}^{\mathbf{b}}$ & $\boldsymbol{\Delta E}+\mathbf{Z P C}^{c}$ & $\mathbf{\Delta H}$ & $\boldsymbol{\Delta G}$ \\
\hline $\mathbf{1}$ & -43.5 & -40.8 & -41.4 & -21.7 \\
\hline $\mathbf{1}^{\prime}$ & -27.8 & -26.0 & -27.0 & -2.6 \\
\hline $\mathbf{2}$ & -43.1 & -40.9 & -41.3 & -22.3 \\
\hline $\mathbf{3}$ & -36.6 & -33.5 & -34.3 & -12.5 \\
\hline $\mathbf{3}^{\prime}$ & -36.7 & -33.6 & -34.4 & -12.8 \\
\hline \multicolumn{5}{|c}{ Experiment } \\
\hline $\mathbf{G P A}$ & $\boldsymbol{K}_{\mathbf{d}}(\boldsymbol{\mu} \mathbf{M})$ & $\mathbf{T} \mathbf{S}$ & $\mathbf{\Delta H}$ & $\boldsymbol{\Delta} \mathbf{G}$ \\
\hline $\mathbf{1}$ & $2.7 \pm 0.4$ & -0.96 & $-8.6 \pm 0.5$ & -7.6 \\
\hline $\mathbf{1}^{\prime}$ & $\sim 20,000$ & - & - & - \\
\hline $\mathbf{2}$ & $1.7 \pm 0.3$ & 0.85 & $-7.0 \pm 0.3$ & -7.9 \\
\hline $\mathbf{3}$ & $2.8 \pm 0.3$ & 0.20 & $-7.4 \pm 0.3$ & -7.6 \\
\hline
\end{tabular}

${ }^{a}$ Chain A was used in all calculations

${ }^{b}$ Electronic binding energies

${ }^{c}$ Zero-point energy correction obtained using frequency calculations. See SI for details.

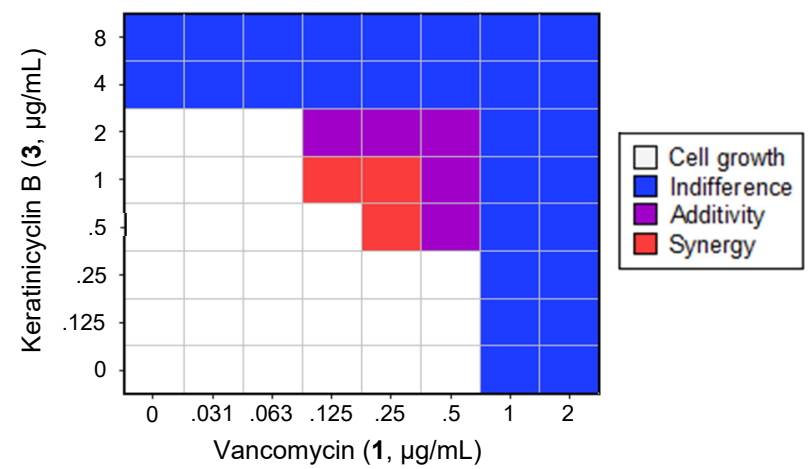

Figure 4. Checkerboard-based synergy analysis with $\mathbf{1}$ and $\mathbf{3}$ against $C$. difficile. See SI and text for details.

In summary, we find that $\mathbf{3}$ is unable to bind the peptidoglycan terminus. Thermodynamic binding studies, crystallographic analysis, and DFT-based computations all suggest that the lack of binding is driven by steric constraints. This conclusion is consistent with prior work in which replacement of D-Ala with bulkier sidechains abolishes binding by $\mathbf{1}$, and with the structures of class IV GPAs, such as teicoplanin, in which a fourth aromatic crosslink between residues 5 and 7 further rigidifies the hydrophobic binding pocket. Our synergy data support the conclusion that $\mathbf{3}$ interferes with an alternative target to inhibit C. difficile growth. Future studies will seek to identify the molecular target of $\mathbf{3}$ and to explore the combination of $\mathbf{1}$ and $\mathbf{3}$ as a viable therapeutic option.

\section{ASSOCIATED CONTENT}

The Supporting Information is available free of charge on the ACS Publications website at:

\section{AUTHOR INFORMATION}




\section{Corresponding Author}

*katherine.davis@emory.edu; mrseyed@princeton.edu

\section{ACKNOWLEDGMENT}

We are grateful to Jamal Musaev and Alex Kaledin at Emory University's Cerry L. Emerson Center for Scientific Computation for assistance with DFT calculations, as well as the Edward C. Taylor $3^{\text {rd }}$ Year Fellowship in Chemistry (to V.T.C.) and the National Institutes of Health (grant 1R00GM129460 to K.M.D. and grant 1R01GM129496 to M.R.S.) for support of this work.

\section{REFERENCES}

1. Barna, J. C. J.; Williams, D. H. The structure and mode of action of glycopeptide antibiotics. Annu. Rev. Microbiol. 1984, 38, 339357.

2. Nicolaou, K. C.; Boddy, C. N.; Bräse, S.; Winssinger, N. Chemistry, Biology, and Medicine of the Glycopeptide Antibiotics. Angew. Chem. Int. Ed. Engl. 1999, 38, 2096-2152.

3. Kahne, D.; Leimkuhler, C.; Lu, W.; Walsh, C. Glycopeptide and lipoglycopeptide antibiotics. Chem. Rev. 2005, 105, 425-448.

4. Okano, A.; Isley, N. A.; Boger, D. L. Total syntheses of vancomycin-related glycopeptide antibiotics and key analogues. Chem. Rev. 2017, 117, 11952-11993.

5. Perkins, H. R. Specificity of combination between mucopeptide precursors and vancomycin or ristocetin. Biochem. J. 1969, 111, 195-205.

6. Nieto, M.; Perkins, H. R. Modifications of the acyl-D-alanyl-Dalanine terminus affecting complex-formation with vancomycin. Biochem. J. 1971, 123, 789-803.

7. Schäfer, M.; Schneider, T. R.; Sheldrick, G. M. Crystal structure of vancomycin. Structure 1996, 4, 1509-1515.

8. Nitanai, Y.; Kikuchi, T.; Kakoi, K.; Hanamaki, S.; Fujisawa, I.; Aoki, K. Crystal structures of the complexes between vancomycin and cell-wall precursor analogs. J. Mol. Biol. 2009, 385, 14221432.

9. Culp, E. J.; Waglechner, N.; Wang, W.; Fiebig-Comyn, A. A.; Hsu, Y.-P.; Koteva, K.; Sychantha, D.; Coombes, B. K.; Van Nieuwenhze, M. S.; Brun, Y. V.; Wright, G. D. Evolution-guided discovery of antibiotics that inhibit peptidoglycan remodelling. Nature 2020, 578, 582-587.

10. Xu, F.; Wu, Y.; Zhang, C.; Davis, K. M.; Moon, K.; Bushin, L. B.; Seyedsayamdost, M. R. A genetics-free method for highthroughput discovery of cryptic microbial metabolites. Nat. Chem. Biol. 2019, 15, 161-168.

11. Rekharsky, M.; Hesek, D.; Lee, M.; Meroueh, S. O.; Inoue, Y.; Mobashery, S. Thermodynamics of interactions of vancomycin and synthetic surrogates of bacterial cell wall. J. Am. Chem. Soc. 2006, 128, 7736-7737.
12. Gerhard, U.; Mackay, J. P.; Maplestone, R. A.; Williams, D. H. The role of the sugar and chlorine substituents in the dimerization of vancomycin antibiotics. J. Am. Chem. Soc. 1993, 115, 232-237.

13. Mackay, J. P.; Gerhard, U.; Beauregard, D. A.; Maplestone, R. A.; Williams, D. H. Dissection of the contributions toward dimerization of glycopeptide antibiotics. J. Am. Chem. Soc. 1994, 116, 4573-4580.

14. Kanna, R.; Harris, C. M.; Harris, T. M.; Waltho, J. P.; Skelton, N. J.; Williams, D. H. Function of the amino sugar and N-terminal amino acid of the antibiotic vancomycin in its complexation with cell well peptides. J. Am. Chem. Soc. 1988, 110, 2946-2953.

15. Booth, P. M.; Williams, D. H. Preparation and conformational analysis of vancomycin hexapeptide and aglucovancomycin hexapeptide. J. Chem. Soc. Perkin Trans. I 1989, 2335-2339.

16. Arthur, M.; Molinas, C.; Bugg, T. D.; Wright, G. D.; Walsh, C. T.; Courvalin, P. Evidence for in vivo incorporation of D-lactate into peptidoglycan precursors of vancomycin-resistant enterococci. Antimicrob. Agents Chemother. 1992, 36, 867-869.

17. Lee, J.; Sagui, C.; Roland, C. First principles investigation of vancomycin and teicoplanin binding to bacterial cell wall termini. $J$. Am. Chem. Soc. 2004, 126, 8384-8385.

18. Lee, J.; Sagui, C.; Roland, C. Quantum simulations of the structure and binding of glycopeptide antibiotic aglycones to cell wall analogues. J. Phys. Chem. 2005, 109, 20588-20596.

19. Becke, A. D. Density-functional thermochemistry. III. The role of exact exchange. J. Chem. Phys. 1993, 98, 5648-5652.

20. Lee, C.; Yang, W.; Parr, R. G. Development of the Colle-Salvetti correlation-energy formula into a functional of the electron density. Phys. Rev. B 1988, 37, 785.

21. Tomasi, J.; Mennucci, B.; Cammi, R. Quantum mechanical continuum solvation models. Chem. Rev. 2005, 105, 2999-3093.

22. Avogadro: an open-source molecular builder and visualization tool. Version 1.2.0. http://avogadro.cc/

23. Hanwell, M. D.; Curtis, D. E.; Lonie, D. C.; Vandermeersch, T.; Zurek, E.; Hutchison, G. R. Avogadro: An advanced semantic chemical editor, visualization, and analysis platform. $J$. Cheminform. 2012, 4, 17.

24. Rappe, A. K.; Casewit, C. J.; Colwell, K. S.; Goddard III, W. A.; Skiff, W. M. UFF, a full periodic table force field for molecular mechanics and molecular dynamics simulation. J. Am. Chem. Soc. 1992, 114, 10024-10035.

25. Orhan, G.; Bayram, A.; Zer, Y.; Balci, I. Synergy test by E test and checkerboard methods of antimicrobial combinations against Brucella melitensis. J. Clin. Microbiol. 2005, 43, 140-143.

26. Tallarida, R. J. An overview of drug combination analysis with isobolograms. J. Pharmacol. Exp. Ther. 2006, 319, 1-7.

27. Wu, Y.; Seyedsayamdost, M. R. Synergy and target promiscuity drive structural divergence in bacterial alkylquinolone biosynthesis. Cell Chem. Biol. 2017, 24, 1437-1444. 
TOC graphic

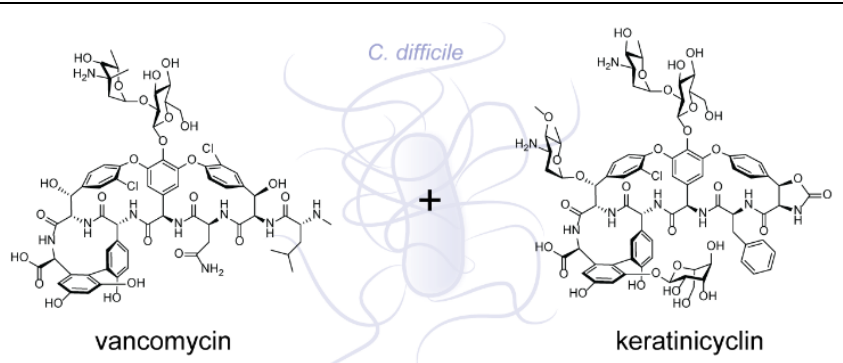

Synergy 